\title{
Comparação entre dois métodos de posicionamento para realização do raio $X$ e sua repercussão na avaliação da cifose torácica utilizando o método de Cobb e no equilíbrio sagital
}

\author{
Comparison between two positioning methods for X-ray and its \\ impact on the evaluation of thoracic kyphosis by Cobb's method and \\ sagittal balance \\ Comparación entre dos métodos de posicionamiento para la \\ realización del rayo $X$ y sus efectos sobre evaluación de la cifosis \\ torácica usando el método de Cobb y el sagital equilibrio
}

\author{
Carlos Henrique Maçaneiro' \\ Ricardo Kiyoshi Miyamoto ${ }^{2}$ \\ Rodrigo Fetter Lauffer ${ }^{2}$ \\ Luciano Leandro Martins ${ }^{3}$
}

\begin{abstract}
RESUMO
Introdução: o tratamento da cifose torácica é baseado na aferição da magnitude da curva baseada em mensurações radiográficas, e proporcionar o correto equilíbrio sagital pela correção cirúrgica de uma gibosidade é de importância fundamental. Objetivo: análise radiográfica prospectiva do efeito da variação da posição dos braços sobre a aferição da cifose torácica e do equilíbrio sagital entre pacientes com idades pré-estabelecidas. Métodos: foram realizadas radiografias na posição lateral da coluna vertebral utilizando-se dois métodos posicionais: posição 1 , em que o paciente permanecia de pé, com os joelhos juntos, pés alinhados com os ombros, cabeça reta direcionada para a frente, braços estendidos a $90^{\circ}$ em relação ao tronco, mantendo os membros su-
\end{abstract}

\section{ABSTRACT}

Introduction: the thoracic kyphosis treatment is based on measuring the magnitude of the curve based on radiographic measurements, and provide the correct balance for the surgical correction of sagittal spinal deformity is very important. Objective: a prospective radiographic analysis of the effect of the variation in arm position on measuring thoracic kyphosis and sagittal balance in patients of a pre-established age. Methods: lateral radiographs of the spine were performed by two positional methods: position 1, in which the patient was standing, with the knees close together, feet aligned with shoulders, head looking straight forward, arms extended at $90^{\circ}$ in relation to the stem, keeping the arms stretched and parallel to the
\end{abstract}

\section{RESUMEN}

Introducción: el tratamiento de la cifosis torácica se basa en la medición de la magnitud de la curva a partir de mediciones radiológicas, y proporcionar el equilibrio adecuado para la corrección quirúrgica de la deformidad de la columna sagital es de una importancia fundamental. Objetivo: un análisis prospectivo radiográfico del efecto de la variación en la posición de los brazos en la medición de la cifosis torácica y el equilibrio sagital en pacientes con edades preestablecidas. Métodos: se realizaron radiografías laterales de la columna vertebral, utilizando dos métodos de posición: posición 1, en la que el paciente estaba de pie, con las rodillas juntas, los pies alineados con los hombros, la cabeza mirando recto hacia adelante, con los brazos extendidos en $90^{\circ}$ con respecto al tronco,

\footnotetext{
Trabalho realizado no Instituto de Ortopedia e Traumatologia do Instituto de Ortopedia e Traumatologia de Joinville - IOT - Joinville (SC), Brasil.

'Chefe do Serviço de Residência em Ortopedia e Traumatologia do Instituto de Ortopedia e Traumatologia de Joinville - IOT - Joinville (SC), Brasil. ${ }^{2}$ Médico do Grupo de Cirurgia da Coluna Vertebral do Instituto de Ortopedia e Traumatologia de Joinville - IOT - Joinville (SC), Brasil.

${ }^{3}$ Residente em Cirurgia da Coluna Vertebral do Instituto de Ortopedia e Traumatologia de Joinville - IOT - Joinville (SC), Brasil.
} 
periores estendidos e paralelos ao chão; uma segunda radiografia foi obtida com o paciente de pé, com os joelhos juntos, pés alinhados com os ombros, cabeça reta direcionada para a frente, com os cotovelos fletidos e os dedos repousando sobre a fossa supraclavicular bilateralmente. Os braços devem formar um ângulo de aproximadamente $45^{\circ}$ com o corpo. Foram mensurados os ângulos de Cobb e o equilíbrio sagital nas duas radiografias. Resultados: não houve correlação entre a posição dos braços, os valores angulares de Cobb e o equilíbrio sagital. Conclusão: em nosso trabalho, observamos que a posição dos braços $\left(90^{\circ}\right.$ versus $\left.45^{\circ}\right)$, não interfere estatisticamente no valor da cifose torácica e na variação do equilíbrio sagital.

\section{DESCRITORES: Cifose/}

radiografia; Raios X; Posicionamento do paciente; Coluna vertebral/radiografia floor; a second radiograph showed the patient standing with knees together, feet aligned with shoulders, head looking straight forward, with the elbows flexed and the fingers resting on the supraclavicular fossa bilaterally. The arms should form an angle of about $45^{\circ}$ with the body. We measured the Cobb angles and the sagittal balance in the two radiographs. Results: there was no correlation between the position of the arms and the values of the Cobb angle and the sagittal balance. Conclusion: in our study, we observed that the position of the arms $\left(90^{\circ}\right.$ versus $\left.45^{\circ}\right)$ statistically does not interfere in the value of thoracic kyphosis and the variation of sagittal balance. manteniendo los brazos extendidos, paralelos al piso; y una segunda radiografía se obtuvo con el paciente de pie con las rodillas juntas, los pies alineados con los hombros, la cabeza mirando recto hacia adelante, con los codos flexionados y los dedos sobre la fosa supra clavicular bilateral. Los brazos deben formar un ángulo de $45^{\circ}$ con el cuerpo. Los ángulos de Cobb y el equilibrio sagital en las dos radiografias fueron mensurados. Resultados: no hubo correlación entre la posición de los brazos, los valores de ángulo de Cobb y el equilibrio sagital. Conclusión: en nuestro estudio, hemos observado que la posición de los brazos $\left(90\right.$ versus $\left.45^{\circ}\right)$, estadisticamente no intervendrá en la determinación de la cifosis torácica y en la variación del equilibrio sagital.
KEYWORDS: Kyphosis/

radiography; X-Rays; Patient positioning; Spine/ radiography

\author{
DESCRIPTORES: Cifosis/ \\ radiografía; Rayos X; \\ Posicionamiento del paciente; \\ Columna vertebral/radiografía
}

\section{INTRODUÇÃO}

$\mathrm{O}$ tratamento da cifose torácica é baseado na magnitude da curva e o curso esperado da história natural em longo prazo. A determinação da cifose torácica normal versus anormal continua sendo motivo de preocupação para o profissional de Medicina ${ }^{1,2}$.

$\mathrm{O}$ valor normal do equilíbrio sagital em adolescentes e adultos assintomáticos tem sido bem descrito ${ }^{2-5}$. Porém, poucos estudos têm analisado a medida da cifose torácica e do equilíbrio sagital envolvendo a posição dos braços do paciente durante a realização do exame radiográfico.

Isso afeta o tratamento quando as decisões são baseadas em mensurações radiográficas e, com as atuais restrições à exposição à radiação, torna-se difícil realizar mais estudos $^{6}$. Proporcionar o correto equilíbrio sagital pela correção cirúrgica de uma gibosidade é de importância primordial ${ }^{7}$.

O objetivo do presente estudo foi o de, prospectivamente, avaliar o efeito da variação da posição dos braços sobre a aferição da cifose torácica e do equilíbrio sagital em pacientes de uma faixa etária pré-estabelecida.

\section{MÉTODOS}

Após ter sido aprovado no Comitê de Ética do Hospital São José, foram colhidas as assinaturas dos termos de consentimento livre e esclarecido. Todos os pacientes foram cadastrados em um protocolo (Anexo 1). A qualquer momento, os participantes poderiam desistir da pesquisa sem que isso causasse nenhum problema.

Foi adotado o nível de significância de 5\% para a aplicação dos testes estatísticos, ou seja, quando a significância calculada (p) fosse menor do que 5\% $(0,050)$, encontrava-se uma diferença (ou relação) estatisticamente significante; quando a significância calculada (p) fosse igual ou maior do que $5 \%(0,050)$, encontrava-se uma diferença (ou relação) estatisticamente não-significante, ou seja, uma semelhança.

Foram incluídos no trabalho pacientes com idade entre 11 e 18 anos, em média 15,20 $( \pm 1,824)$, perfazendo um $n$ total de 20 pessoas. Desse total, 14 eram do sexo masculino e 6 do sexo feminino. As médias dos valores do ângulo de Cobb na posição 1 e 2 foram, respectivamente, $58,55^{\circ}$ e $58,65^{\circ}$. Os valores estão demonstrados nas tabelas 1,2 e 3 .

Uma primeira radiografia lateral (posição 1) foi obtida com o paciente de pé, joelhos juntos, pés alinhados com os ombros, cabeça reta direcionada para a frente, braços estendidos a $90^{\circ}$ em relação ao tronco, mantendo os membros superiores estendidos, paralelos ao chão e sem apoio.

Uma segunda radiografia lateral (posição 2) foi obtida com o paciente de pé, joelhos juntos, pés alinhados com os ombros, cabeça reta direcionada para a frente, cotovelos 
TABELA 1 - Distribuição dos pacientes em relação ao sexo

\begin{tabular}{lcccc}
\hline Sexo & Frequência & $\%$ & Porcentagem válida & Porcentagem cumulativa \\
\hline Masculino & 14 & 70,0 & 70,0 & 70,0 \\
Feminino & 6 & 30,0 & 30,0 & 100,0 \\
Total & 20 & 100,0 & 100,0 & \\
\hline
\end{tabular}

TABELA 2 - Valores obtidos nas posições 1 e 2

\begin{tabular}{lccc}
\hline & Posição 1[POS1] & Posição 1[POS2] & Valor p \\
\hline Ângulo de Cobb [COBB] & $58,55(17,69)$ & $58,65(16,95)$ & $>0,05$ \\
Balanço Sagital [SAGB] & & & $>0,05$ \\
+ & $20,0 \%$ & $20,0 \%$ & \\
0 & $55,0 \%$ & $55,0 \%$ & \\
- & $25,0 \%$ & $25,0 \%$ & \\
\hline
\end{tabular}

TABELA 3 - Média dos valores angulares de Cobb obtidos nos dois métodos

\begin{tabular}{cccccc}
\hline & & Média & $\mathbf{n}$ & Desvio padrão & Erro padrão da diferença média \\
\hline Par 1 & COBB 1 & 58,65 & 20 & 17,695 & 3,957 \\
& COBB2 & 58,65 & 20 & 16,956 & 3,791 \\
\hline
\end{tabular}

fletidos e dedos repousando sobre a fossa supraclavicular bilateralmente. Os braços devem formar um ângulo de aproximadamente $45^{\circ}$ com o corpo (Figuras $1 \mathrm{~A} \mathrm{e} \mathrm{B}$ ).

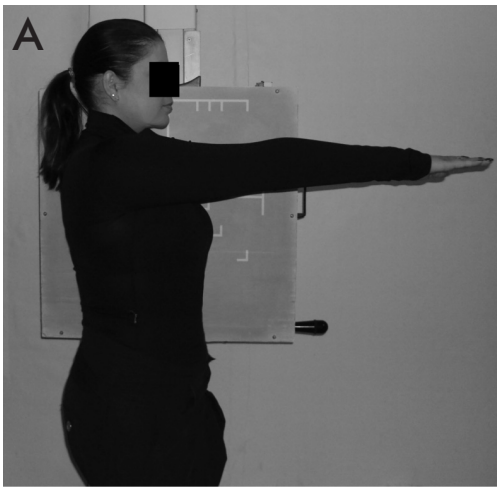

Figura 1

Paciente na posição 1 e na posição 2 para realização do exame radiográfico.

A radiografia foi centrada na sétima vértebra torácica e realizada após completa expiração.

A cifose torácica foi mensurada utilizando-se o método de Cobb por meio da placa terminal superior de T3 e a placa terminal inferior de T12 (Figura 2).

A distância na horizontal entre as partes posterior e superior do sacro ao prumo linha foi registrada como a magnitude do eixo sagital vertical (SVA) em centímetros. Um valor neutro do equilíbrio sagital foi indicado por uma linha que passa sobre o sacro. Um valor positivo do equilíbrio sagital indica que a linha de prumo passa na frente do sacro, e um valor negativo indica que a linha passa atrás do sacro (Figura 3).

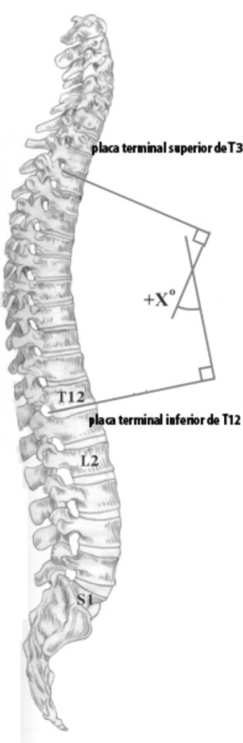

Figura 2

Avaliação da cifose torácica utilizando o método de Cobb.

Todos os pacientes foram selecionados dentre os pacientes dos três cirurgiões. Todos foram voluntários e preencheram os critérios de inclusão: idade entre 10 e 20 anos, ausência de escoliose, fraturas ou cirurgias prévias da coluna vertebral.

Todos os filmes foram mensurados por um único investigador (CHM).

\section{RESULTADOS}

Os resultados mostraram que não houve relação estatisticamente significativa $(p>0,05)$ entre a posição dos braços e a aferição do ângulo de Cobb e do equilíbrio sagital nas posições 1 e 2 (Tabelas 2, 3 e 4). Para melhor visualização, foram colocados gráficos para demonstrar os achados das medições (Gráficos 1 e 2). 


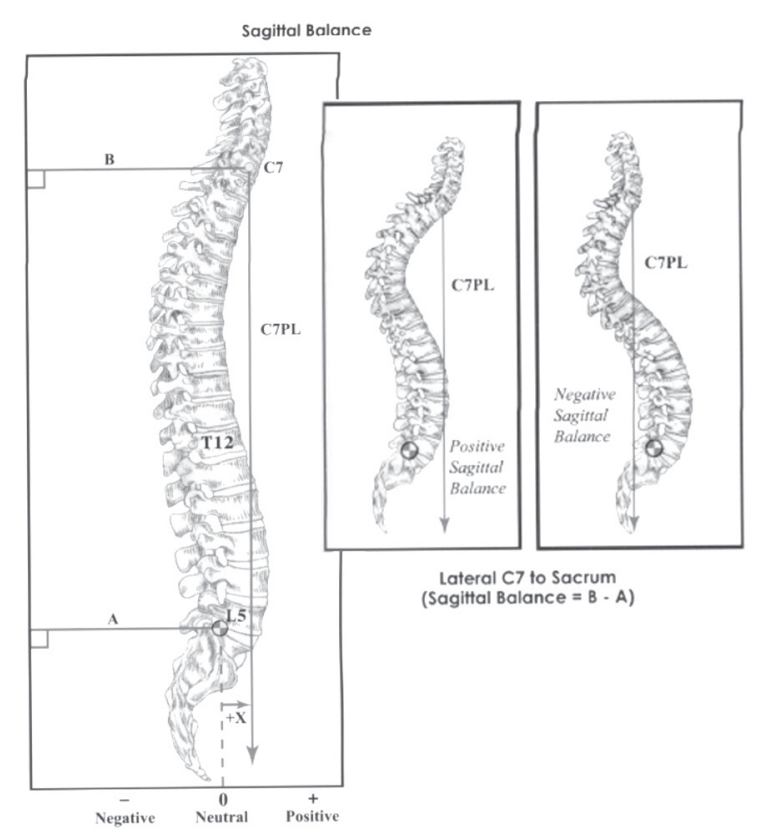

Figura 3

Avaliação do equilíbrio sagital.

\section{DISCUSSÃO}

Grandes amostras permitem conhecer com mais propriedade o que ocorre na população. Porém, manter o tamanho da amostra em um mínimo necessário é conveniente por questões práticas e financeiras e, às vezes, até por implicações éticas, como nos casos de produtos potencialmente perigosos à saúde. Porém, como este foi um estudo que exigiu muitas radiografias, tornou-se muito difícil fazer o trabalho com muitos indivíduos ${ }^{8}$.

Kado et al. realizaram um estudo comparando vários métodos de mensuração da cifose torácica em pacientes idosos, tanto clínicos quanto radiológicos, e demonstraram que a aferição por meio do método digital é mais acurada do que a do método manual ${ }^{9}$.

Poucos estudos têm avaliado a correlação entre as diferentes mensurações da cifose torácica, e a maioria tem sido realizada na população jovem ${ }^{10-15}$.

Modernos sistemas segmentares de instrumentação vertebral têm a capacidade de alterar significativamente os alinhamentos regional e segmentar e o equilíbrio global da coluna vertebral. Por conseguinte, é importante definir não só os valores normais do alinhamento angular vertebral da

\section{TABELA 4 - Distribuição do equilíbrio sagital}

\begin{tabular}{|c|c|c|c|c|c|}
\hline & & & \multicolumn{3}{|c|}{ Posição } \\
\hline & & & Posição 1 & Posição 2 & Total \\
\hline \multirow[t]{8}{*}{ SAGB } & -1 & Contagem \% dentro da posição & 4 & 4 & 8 \\
\hline & & & $20,0 \%$ & $20,0 \%$ & $20,0 \%$ \\
\hline & 0 & Contagem \% dentro da posição & 11 & 11 & 22 \\
\hline & & & $55,0 \%$ & $55,0 \%$ & $55,0 \%$ \\
\hline & 1 & Contagem \% dentro da posição & 5 & 5 & 10 \\
\hline & & & $25,0 \%$ & $25,0 \%$ & $25,0 \%$ \\
\hline & Total & Contagem \% dentro da posição & 20 & 20 & 40 \\
\hline & & & $100,0 \%$ & $100,0 \%$ & $100,0 \%$ \\
\hline
\end{tabular}

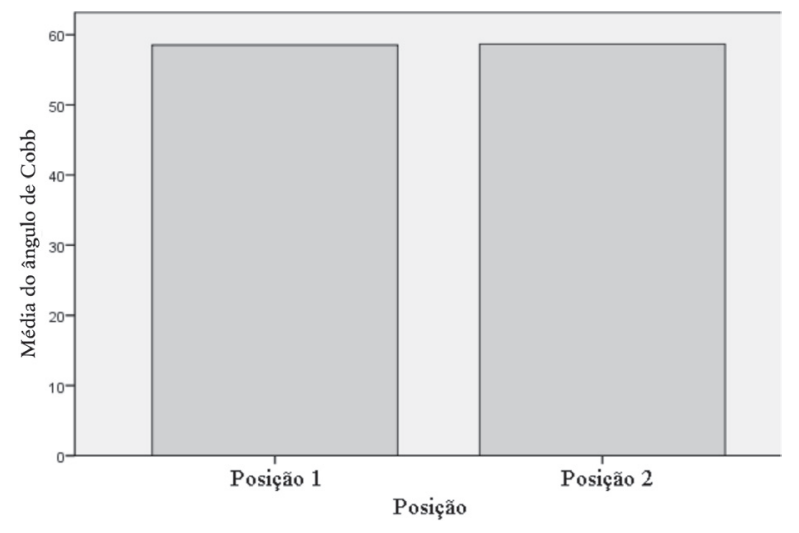

Gráfico 1

Relação entre ângulo de Cobb e as posições 1 e 2

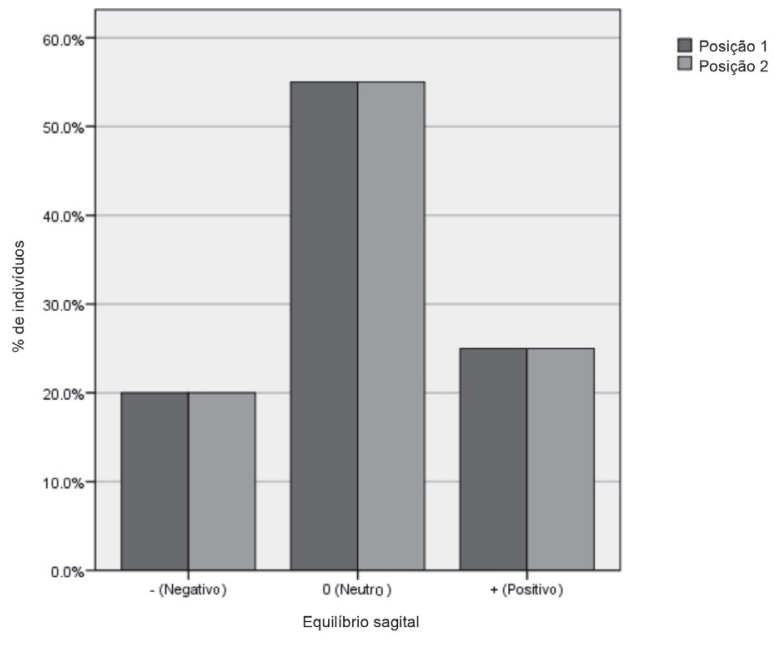

Gráfico 2

Relação entre o equilíbrio sagital e as posições 1 e 2 
coluna, mas também o alinhamento global e o equilíbrio sagital da coluna vertebral ${ }^{16,17}$.

Existe um aumento do reconhecimento da importância do contorno sagital na função normal da coluna e nos seus vários estados patológicos. Por isso, é importante realizar radiografias de uma forma padronizada.

É possível que os diversos grupos musculares do tronco e das extremidades, de alguma forma, desempenhem um papel no controle do alinhamento sagital espinhal, e isso pode interferir na aferição do contorno sagital dependendo da posição adotada pelo paciente de pé no momento de realizar o exame radiográfico ${ }^{18}$.

Uma posição ideal para realização da radiografia lateral da coluna, seria com o paciente em pé, e com os braços descansados ao lado do corpo. No entanto, isto obviamente iria obstruir a visão lateral da coluna vertebral.

Stagnara et al. mencionaram em seu trabalho que a posição dos braços em linha reta ou acima da cabeça tem demonstrado alterações consideráveis no resultado da cifose e da lordose vertebral ${ }^{19}$.

Vedantam et al. compararam no seu estudo a realização de radiografias com os braços na posição $90^{\circ}$ versus $30^{\circ}$, e chegaram à conclusão de que a posição dos braços a $90^{\circ}$ resultou em um equilíbrio sagital negativo naqueles pacientes que apresentavam fusão vertebral prévia ${ }^{18}$. Por isso, esses autores recomendam o posicionamento dos braços na posição de $30^{\circ}$ em relação à vertical do corpo para otimizar e padronizar exames radiográficos da coluna lateral.
Boseker et al. realizaram um estudo com 121 crianças, sem patologia vertebral, para avaliação do valor angular da cifose torácica, e citam no seu trabalho a importância da determinação da cifose torácica normal versus anormal: a variação do valor angular devido à posição dos braços e a disponibilidade de poucos estudos, principalmente devidos às restrições impostas pela exposição às radiações ${ }^{20}$.

Marks et al. observaram em um estudo envolvendo 15 voluntárias femininas que, quando estavam com $45^{\circ}$ de flexão dos ombros, havia uma mudança negativa no equilíbrio sagital de cerca de $6 \mathrm{~cm}$ em relação à posição de relaxamento. Essa mudança foi acompanhada por rotação leve posterior da pelve, embora não estatisticamente significativa, à extensão dos quadris ${ }^{6}$.

Em nosso estudo, realizado com pacientes jovens, a posição dos braços tanto em $45^{\circ}$ de flexão dos ombros como em linha reta, paralelos ao chão, não afetou de maneira significativa a avaliação da cifose torácica e do equilíbrio sagital.

\section{CONCLUSÕES}

Em nosso estudo, observamos que a posição dos braços $\left(90^{\circ}\right.$ versus $\left.45^{\circ}\right)$ não interfere estatisticamente no valor da cifose torácica e na variação do equilíbrio sagital no grupo estudado. Ressaltamos a importância de realizar trabalhos que envolvam pacientes de outros grupos etários e também trabalhos de âmbito multicentro para melhor avaliação desses métodos.

\section{REFERÊNCIAS}

1. Fon GT, Pitt MJ, Thies AC Jr. Thoracic kyphosis: range in normal subjects. AJR Am J Roentgenol. 1980;134(5):979-83.

2. Vedantam R, Lenke LG, Keeney JA, Bridwell KH. Comparison of standing sagittal spinal alignment in asymptomatic adolescents and adults. Spine (Phila Pa 1976). 1998;23(2):211-5.

3. Bernhardt M, Bridwell KH. Segmental analysis of the sagittal plane alignment of the normal thoracic and lumbar spines and thoracolumbar junction. Spine (Phila Pa 1976). 1989;14(7):717-21.

4. Gelb DE, Lenke LG, Bridwell KH. Blanke K, McEnery KW. An analysis of sagittal spinal alignment in 100 asymptomatic middle and older aged volunteers. Spine (Phila Pa 1976). 1995;20(12):1351-8.
5. Jackson RP, McManus AC. Radiographic analysis of sagittal plane alignment and balance in standing volunteers and patients with low back pain matched for age, sex, and size: a prospective controlled clinical study. Spine (Phila Pa 1976). 1994;19(14):1611-8.

6. Marks MC, Stanford CF, Mahar AT, Newton PO. Standing lateral radiographic positioning does not represent customary standing balance. Spine (Phila Pa 1976). 2003;28(11):1176-82.

7. Vialle R, Levassor N, Rillardon L, Templier A, Skalli W, Guigui P. Radiographic analysis of the sagittal alignment and balance of the spine in asymptomatic subjects. J Bone Joint Surg Am. 2005;87(2):260-7.

8. Pereira M. Epidemiologia: teoria e prática. $3^{\text {a }}$ ed. Rio de Janeiro: Guanabara Koogan; 1995. p. 358-76.
9. Kado MD, Christianson L, Palermo L, Smith-Bindman R, Cummings SR, Greendale GA. Comparing a supine radiologic versus standing clinical measurement of kyphosis in older women: the Fracture Intervention Trial. Spine (Phila Pa 1976). 2006;31(4):463-7.

10.McAlister WH, Shackelford GD. Measurement of spinal curvatures. Radiol Clin North Am. 1975;13(1):113-21.

11.O'Brien MF, Lenke LG. Radiographic measurement manual. USA: Medtronic Sofamor Danek; 2005.

12.Stotts AK, Smith JT, Santora SD, Roach JW, D'Astous JL. Measurement of spinal kyphosis: implications for the management of Scheuermann's kyphosis. Spine (Phila Pa 1976). 2002;27(19):2143-6.

13.Korovessis P, Petsinis G, Papazisis Z, Baikousis A. Prediction of thoracic kyphosis using the Debrunner kyphometer. J Spinal Disord. 2001;14(1):67-72. 
14.Goh S, Price RI, Leedman PJ, Singer KP. A comparison of three methods for measuring thoracic kyphosis: implications for clinical studies. Rheumatology (Oxford). 2000;39(3):310-5.

15.Lundon KM, Li AM, Bibershtein S. Interrater and intrarater reliability in the measurement of kyphosis in postmenopausal women with osteoporosis. Spine (Phila Pa 1976). 1998;23(18):1978-85.

16.Voutsinas SA, MacEwen GD. Sagittal profiles of the spine. Clin Orthop Relat Res. 1986;(210):235-42.
17.Lenke LG, Bridwell KH, Blanke K, Baldus C, Weston J. Radiographic results of arthrodesis with CotrelDubousset instrumentation for the treatment of adolescent idiopathic scoliosis. A five to ten-year followup study. J Bone Joint Surg Am. 1998;80(6):807-14.

18. Vedantam R, Lenke LG, Bridwell KH, Linville DL, Blanke K. The effect of variation in arm position on sagittal spinal alignment. Spine (Phila $\mathrm{Pa}$ 1976).2000; 25(17): 2204-9.

19.Stagnara P, De Mauroy JC, Dran G, Gonon GP, Costanzo G, Dimnet J, et al. Reciprocal angulation of vertebral bodies in a sagittal plane: approach to references in the evaluation of kyphosis and lordosis. Spine (Phila $\mathrm{Pa}$ 1976). 1982;7(4):335-42.
20.Boseker EH, Moe JH, Winter RB, Koop SE. Determination of "normal" thoracic kyphosis: a roentgenographic study of 121 "normal" children. J Pediatr Orthop. 2000;20(6):796-8.

\section{Correspondência}

Carlos Henrique Maçaneiro

Rua Blumenau, 1.316

CEP: 89204-251 - Joinville (SC), Brasil

E-mail: iot@iot.com.br 


\section{ANEXO 1}

\section{INSTITUTO DE ORTOPEDIA E TRAUMATOLOGIA \\ CIRURGIA DA COLUNA JOINVILLE - SC \\ RESIDENTE: LUCIANO LEANDRO MARTINS \\ ORIENTADOR: DR. CARLOS HENRIQUE MAÇANEIRO \\ PROTOCOLO SOBRE O TRABALHO ENTITULADO “COMPARAÇÃO ENTRE DOIS MÉTODOS DE POSICIONAMENTO PARA REALIZAÇÃO DO RAIO-X E SUA REPERCUSSÃO NA AVALIAÇÃO DA CIFOSE TORÁCICA UTILIZANDO O MÉTODO DE COBB E NO BALANÇO SAGITAL"}

FICHA DE IDENTIFICAÇÃO

PRONTUÁRIO

NOME:

IDADE:

PROFISSÃO:

SEXO I MU I) F

ALTURA:

PESO:

TELEFONE:

PATOLOGIA VERTEBRAL:

Objetivos

O trabalho tem como objetivo comparar dois métodos posicionamento para realização do raio-x de perfil da coluna e averiguar se há diferença estatisticamente significativa da cifose torácica aferida pelo método de Cobb e alteração no equilíbrio sagital devido as alteração nas posições do braços.

ÁNALISE

\section{POSIÇÃO 1 [POS1]}

Paciente em pé, com os joelhos juntos, pés alinhados com os ombros, com a cabeça reta olhando para frente, com os braços estendidos a $90^{\circ}$ em relação ao tronco, mantendo os membros superiores estendidos, paralelos ao chão.

Valor angular da cifose torácica aferida pelo método de Cobb [COBB 1 ]:

Vértebras terminais superior e inferior:

Balanço Sagital [SAGB 1]:

\section{POSIÇÃO 2 [POS2]}

Paciente em pé, com os joelhos juntos, pés alinhados com os ombros, com a cabeça reta olhando para frente, com os cotovelos fletidos e os dedos repousando sobre a fossa supraclavicular bilateralmente. Os braços devem formar um ângulo de aproximadamente $45^{\circ} \mathrm{com}$ o corpo.

Valor angular da cifose torácica aferida pelo método de Cobb [COBB2]

Vértebras terminais superior e inferior:

Balanço Sagital [SAGB2]:

Fatores técnicos:

Tamanho do filme: $35 \times 43 \mathrm{~cm}$

Grade móvel. Filtro de compensação. Dois técnicos treinados.

Posicionar o paciente de modo que a coluna vertebral esteja paralela ao filme.

Raio Central perpendicular ao eixo longo da coluna vertebral, centralizado em T7, que está 3 a 4 polegadas abaixo da incisura jugular, ou 1 a 2 polegadas abaixo do ângulo esternal, em um paciente adulto médio.

Realizado colimação nas margens para expor um campo de 5 a 6 polegadas de largura, com bordas superior e inferior em direção às margens do filme. Distância do paciente $x$ aparelho:

O paciente deve prender a respiração após a expiração completa. Assegurado que não há rotação da pelve ou dos ombros. Visualização de todas as vértebras até o sacro.

Joinville, 